\title{
Synchronization of the Coupled Distributed Parameter System with Time Delay via Proportional-Spatial Derivative Control
}

\author{
Kun Yuan, ${ }^{1}$ Abdulaziz Alofi, ${ }^{2}$ Jinde Cao, ${ }^{1,2}$ Abdullah Al-Mazrooei, ${ }^{2}$ and Ahmed Elaiw ${ }^{2}$ \\ ${ }^{1}$ School of Automation and Key Laboratory of Measurement and Control of Complex Systems of Engineering, \\ Ministry of Education, Southeast University, Nanjing 210096, China \\ ${ }^{2}$ Department of Mathematics, Faculty of Science, King Abdulaziz University, Jeddah 21589, Saudi Arabia
}

Correspondence should be addressed to Kun Yuan; kyuan@seu.edu.cn

Received 16 December 2013; Revised 27 January 2014; Accepted 9 February 2014; Published 16 March 2014

Academic Editor: Wei Lin

Copyright (C) 2014 Kun Yuan et al. This is an open access article distributed under the Creative Commons Attribution License, which permits unrestricted use, distribution, and reproduction in any medium, provided the original work is properly cited.

By combining parabolic partial differential equation (PDE) theory with Lyapunov technique, the synchronization is studied for a class of coupled distributed parameter systems (DPS) described by PDEs. First, based on Kronecker product and Lyapunov functional, some easy-to-test sufficient condition is given to ensure the synchronization of coupled DPS with time delay. Secondly, in the case that the whole coupled system cannot synchronize by itself, the proportional-spatial derivative (P-sD) state feedback controller is designed and applied to force the network to synchronize. The sufficient condition on the existence of synchronization controller is given in terms of a set of linear matrix inequalities. Finally, the effectiveness of the proposed control design methodology is demonstrated in numerical simulations.

\section{Introduction}

Most practical systems are distributed in space and time, for example, systems related to heat flows, fluid flow, or flexible structure. The systems are called distributed parameter systems (DPSs), which are mathematically modelled by partial differential equations (PDEs) with boundary conditions. The key characteristic of DPSs is that their inputs, outputs, process states, and the relevant parameters may vary temporally as well as spatially. Due to such characteristic and infinite dimensional, the distributed parameter system is difficult to control. Recent research [1-12] show that the dynamical behavior of the parabolic PDEs can be described approximately in an array of low-order ordinary differential equations (ODEs). In [3], by integrating the Galerkin method with neural networks, the author proposes a simple and effective modeling method for DPS. In [13, 14], the K-L method is applied to model the distributed parameter system. In [4], by the Galerkin's method and the geometric control, the authors stabilize DPSs. For nonlinear ODE system, fuzzymodel-based control technique is conceptually simple and effective. Recently, it has been successfully applied to fuzzy control design of nonlinear DPSs [15-20]. Despite these results, the control of distributed parameter systems is a broad area of research, and further results are demanded to design an effective control method, which motivates our study.

The coupled system can be regarded as a large set of interconnected nodes, which can be expressed by the graph. Each node of the graph represents individual, and the edge of the graph represents the connections among them. The dynamical analysis of coupled system has become a focal topic of great interest, particularly the synchronization phenomena. Synchronization in an array of coupled dynamical systems was first investigated in [21]. Later, some results on synchronization in various coupled systems have been given in [22-26]. It is noted that the dynamical behavior of a coupled system is determined not only by the dynamical behaviors of the isolated nodes, but also by the edges, which depends on the coupled topology of the system. As a special case of coupled system, coupled distributed parameter systems have been an interest. If the coupled DPS is regarded as the special graph, the nodes of the network are linear DPS, the edges of the network are parabolic spatial differential operator with diffusive coupling. In other words, if every node is thermal process, the edges represent the thermal transmittance among the nodes. Synchronization of coupled 
system has been investigated by some researchers [27], and some synchronization criteria are given.

In the case that the whole coupled system cannot synchronize by itself, some controllers should be designed and applied to force the network to synchronize. Due to the fact that the outputs, inputs, process states, and the relevant parameters of DPS may vary temporally as well as spatially, a special control strategy called proportional-spatial derivative (P-sD) control [17] is designed to achieve synchronization of coupled DPS, which is similar as the traditional PD (proportional-time derivative) control.

Motivated by the above discussion, the aim of this paper is to synchronize coupled distributed parameter systems with time delay. First, based on Kronecker product and Lyapunov functional, some easy-to-test sufficient condition is given to ensure the synchronization of coupled DPS with time delay. Secondly, in the case that the whole coupled system cannot synchronize by itself, the proportional-spatial derivative ( $\mathrm{P}$ $\mathrm{sD})$ state feedback controller is designed and applied to force the network to synchronize. The sufficient condition on the existence of synchronization controller is given in terms of a set of linear matrix inequalities. Finally, a numerical example is given to show effectiveness of the proposed method.

Notation. Throughout this paper, for real symmetric matrices $X$ and $Y$, the notation $X \geq Y$ (resp., $X>Y$ ) means that the matrix $X-Y$ is positive semidefinite (resp., positive definite). The superscript " $T$ " represents the transpose. Matrix dimensions, if not explicitly stated, are assumed to be compatible for algebraic operations.

\section{Model Description and Preliminaries}

Consider a dynamical network consisting of $N$ identical and diffusively coupled nodes, with each node being an $n$ dimensional delayed distributed parameter system. The state equations of the network are

$$
\begin{aligned}
\frac{\partial T_{k}}{\partial t}= & D \frac{\partial^{2} T_{k}}{\partial z^{2}}+A T_{k}(t, z)+B T_{k}(t-\tau, z) \\
& +\sum_{j=1, j \neq k}^{N} G_{k j} \frac{\partial^{2}\left(T_{j}-T_{k}\right)}{\partial z^{2}}, \\
& z \in\left(z_{1}, z_{2}\right), \quad k=1,2, \ldots, N
\end{aligned}
$$

subject to the boundary conditions

$$
\begin{aligned}
& \left.\frac{\partial T_{k}(t, z)}{\partial t}\right|_{z=z_{1}}=0, \\
& \left.\frac{\partial T_{k}(t, z)}{\partial t}\right|_{z=z_{2}}=0
\end{aligned}
$$

and the initial condition

$$
T_{k}(t, z)=\phi_{k}(t, z), \quad t \in[-\tau, 0],
$$

where $z \in\left[z_{1}, z_{2}\right]$ is the spatial coordinate, $T_{k}(z, t):\left[z_{1}, z_{2}\right] \times$ $\mathbb{R} \rightarrow \mathbb{R}^{n}$ denotes state variables, $k=1,2, \ldots, N$ is positive constant, and $\tau>0$ denotes the state delay; the vector function $f(\cdot)$ and $g(\cdot)$ are nonlinear locally Lipschitz continuous functions. $G=\left(G_{i j}\right)_{N \times N}$ is the coupling configuration matrix representing the topological structures of the networks which satisfies the diffusive coupling connection:

$$
G_{i j} \geq 0, \quad i \neq j, \quad G_{i i}=-\sum_{j=1, j \neq i}^{N} G_{i j},
$$

which ensures the diffusion that $\sum_{j=1}^{N} G_{i j}=0$.

Equivalently, system (1) can be rewritten in a form as follows:

$$
\begin{aligned}
\frac{\partial T_{k}}{\partial t}= & D \frac{\partial^{2} T_{k}}{\partial z^{2}}+A T_{k}(t, z)+B T_{k}(t-\tau, z) \\
+ & \sum_{j=1}^{N} G_{k j} \frac{\partial^{2} T_{j}}{\partial z^{2}}, \\
& \quad z \in\left(z_{1}, z_{2}\right), \quad k=1,2, \ldots, N .
\end{aligned}
$$

Let $T(t, z)$ be a function to which all $T_{k}(t, z)$ are expected to synchronize and $T(t, z)$ satisfies the following equation:

$$
\frac{\partial T}{\partial t}=D \frac{\partial^{2} T}{\partial z^{2}}+A T(t, z)+B T(t-\tau, z), \quad z \in\left(z_{1}, z_{2}\right)
$$

subject to the boundary conditions

$$
\begin{aligned}
& \left.\frac{\partial T(t, z)}{\partial t}\right|_{z=z_{1}}=0 \\
& \left.\frac{\partial T(t, z)}{\partial t}\right|_{z=z_{2}}=0
\end{aligned}
$$

and the initial condition

$$
T(t, z)=\phi(t, z), \quad t \in[-\tau, 0] .
$$

Our objective is to investigate the synchronization of network (1) with respect to the isolated node $T(t, z)$. Denote $e_{i}(t, z)=T_{i}(t, z)-T(t, z)$; then the following error dynamical system is obtained:

$$
\begin{aligned}
\frac{\partial e_{k}}{\partial t}= & D \frac{\partial^{2} e_{k}}{\partial z^{2}}+A e_{k}(t, z)+B e_{k}(t-\tau, z) \\
+ & \sum_{j=1}^{N} G_{k j} \frac{\partial^{2} e_{j}}{\partial z^{2}}, \\
& z \in(0, l), \quad k=1,2, \ldots, N .
\end{aligned}
$$

The error system is subject to the boundary conditions and initial values as follows:

$$
\begin{aligned}
& \left.\frac{\partial e_{k}(t, z)}{\partial t}\right|_{z=z_{1}}=0 \\
& \left.\frac{\partial e_{k}(t, z)}{\partial t}\right|_{z=z_{2}}=0
\end{aligned}
$$


and the initial condition

$$
e_{k}(t, z)=\phi_{k}(t, z)-\phi(t, z), \quad t \in[-\tau, 0]
$$

Suppose $\mathbf{e}(t, z)=\left[e_{1}^{T}(t, z), e_{2}^{T}(t, z), \ldots, e_{N}^{T}(t, z)\right]^{T} \in R^{n N}$; then the error system (9) can be written as

$$
\begin{aligned}
\frac{\partial \mathbf{e}}{\partial t}= & \left(I_{N} \otimes D\right) \frac{\partial^{2} \mathbf{e}}{\partial z^{2}}+\left(I_{N} \otimes A\right) \mathbf{e}(t, z) \\
& +\left(I_{N} \otimes B\right) \mathbf{e}(t-\tau, z) \\
& +\left(G \otimes I_{n}\right) \frac{\partial^{2} \mathbf{e}}{\partial z^{2}}, \quad z \in\left(z_{1}, z_{2}\right),
\end{aligned}
$$

where $\otimes$ denotes the Kronecker product of the two matrices.

Definition 1. The coupled distributed parameter system in (1) is asymptotically synchronous if the synchronization error satisfies $\lim _{t \rightarrow \infty} e_{k}(t, z)=0$ for all $z \in\left[z_{1}, z_{2}\right]$ and $k=$ $1,2, \ldots, N$.

Let $\bar{D}=I_{N} \otimes D+G \otimes I_{n}, \bar{A}=I_{N} \otimes A$ and $\bar{B}=I_{N} \otimes B$; the system (12) can be rewritten as follows:

$$
\frac{\partial \mathbf{e}}{\partial t}=\bar{D} \frac{\partial^{2} \mathbf{e}}{\partial z^{2}}+\bar{A} \mathbf{e}(t, z)+\bar{B} \mathbf{e}(t-\tau, z)
$$

$\bar{A} \in \mathbb{R}^{n N \times n N}, \bar{B} \in \mathbb{R}^{n N \times n N}$.

Theorem 2. For the coupled DPS (5), synchronize to the system (6) if there are matrices $P=P^{T}>0, Q=Q^{T}>0$, satisfying the following linear matrix inequalities:

$$
\Omega=\left[\begin{array}{ccc}
P \bar{A}+\bar{A}^{T} P+Q & P \bar{B} & 0 \\
\bar{B}^{T} P & -Q & 0 \\
0 & 0 & -2 P \bar{D}
\end{array}\right]<0 .
$$

Consider the following Lyapunov functional candidate for system

$$
\begin{aligned}
V(t)= & \int_{z_{1}}^{z_{2}} \mathbf{e}^{T}(t, z) P \mathbf{e}(t, z) d z \\
& +\int_{z_{1}}^{z_{2}} \int_{t-\tau}^{t} \mathbf{e}^{T}(\theta, z) Q \mathbf{e}(\theta, z) d \theta d z, \\
\frac{d V}{d t}= & 2 \int_{z_{1}}^{z_{2}} \mathbf{e}^{T}(t, z) P \mathbf{e}_{t}(t, z) d z \\
& +\int_{z_{1}}^{z_{2}}\left[\mathbf{e}^{T}(t, z) Q \mathbf{e}(t, z)-\mathbf{e}^{T}(t-\tau, z)\right.
\end{aligned}
$$

$$
\begin{gathered}
\times Q \mathbf{e}(t-\tau, z)] d z \\
+2 \int_{z_{1}}^{z_{2}} \mathbf{e}^{T}(t, z) P\left[\bar{D} \mathbf{e}_{z z}(t, z) d z+\bar{A} \mathbf{e}(t, z)\right. \\
+\bar{B} \mathbf{e}(t-\tau, z)] d z \\
+\int_{z_{1}}^{z_{2}}\left[\mathbf{e}^{T}(t, z) Q \mathbf{e}(t, z)\right. \\
+\int_{z_{1}}^{z_{2}} \mathbf{e}^{T}(t, z) P \bar{D} \mathbf{e}_{z z}(t, z) d z \\
+\int_{z_{1}}^{z_{2}} \mathbf{e}^{T}(t, z)[2 P \bar{A}+Q] \mathbf{e}(t, z) d z \\
+\int_{z_{1}}^{z_{2}} \mathbf{e}^{T}(t, z) 2 P \bar{B} \mathbf{e}(t-\tau, z) d z \\
+\int_{z_{1}}^{z_{2}} \mathbf{e}^{T}(t-\tau, z) Q \mathbf{e}(t-\tau, z) d z .
\end{gathered}
$$

From integrating by parts and taking into account (10), we have

$$
\begin{aligned}
2 \int_{z_{1}}^{z_{2}} e^{T}(t, z) P \bar{D} \mathbf{e}_{z z}(t, z) d z \\
=2 \int_{z_{1}}^{z_{2}} e^{T}(t, z) P \bar{D} d\left(e_{z}(t, z)\right) \\
=\left.2 e^{T}(t, z) P \bar{D} e_{z}(t, z)\right|_{z_{1}} ^{z_{2}} \\
\quad-2 \int_{z_{1}}^{z_{2}} e_{z}^{T}(t, z) P \bar{D} e_{z}(t, z) d z \\
=-2 \int_{z_{1}}^{z_{2}} e_{z}^{T}(t, z) P \bar{D} e_{z}(t, z) d z
\end{aligned}
$$

Using (26) and (16), we find that

$$
\begin{gathered}
\frac{d V}{d t}=\int_{z_{1}}^{z_{2}} \xi^{T}(t, z) \Omega \xi(t, z) d z, \\
\text { where } \xi(t, z)=\left[\mathbf{e}^{T}(t, z), \mathbf{e}^{T}(t-\tau, z), \mathbf{e}_{z}^{T}(t, z)\right]^{T} \text { and } \\
\Omega=\left[\begin{array}{ccc}
P \bar{A}+\bar{A}^{T} P+Q & P \bar{B} & 0 \\
\bar{B}^{T} P & -Q & 0 \\
0 & 0 & -2 P \bar{D}
\end{array}\right] .
\end{gathered}
$$




\section{Synchronization Control}

If the coupled distributed parameter system cannot synchronize to the isolate node (6), the controller will be designed. Consider

$$
\begin{aligned}
\frac{\partial T_{k}}{\partial t}= & D \frac{\partial^{2} T_{k}}{\partial z^{2}}+A T_{k}(t, z)+B T_{k}(t-\tau, z) \\
& +\sum_{j=1, j \neq k}^{N} G_{k j} \frac{\partial^{2}\left(T_{j}-T_{k}\right)}{\partial z^{2}}+C u(t, z), \\
& z \in\left(z_{1}, z_{2}\right), k=1,2, \ldots, N, \\
\frac{\partial \mathbf{e}}{\partial t}= & \bar{D} \frac{\partial^{2} \mathbf{e}}{\partial z^{2}}+\bar{A} \mathbf{e}(t, z)+\bar{B} \mathbf{e}(t-\tau, z) \\
& +\bar{C} u(t, z), \quad i \in S \triangleq 1,2, \ldots, r,
\end{aligned}
$$

where $\bar{C}=I_{n} \otimes C$, and

$$
u(t, z)=K \mathbf{e}(t, z)+L \mathbf{e}_{z}(t, z),
$$

where $K$ and $L$ are $m \times n N$ gain matrices to be determined.

With the control law (21), the overall close-loop system can be written as

$$
\begin{aligned}
\mathbf{e}_{t}(t, z)= & \bar{D} \mathbf{e}_{z z}(t, z)+(\bar{A}+\bar{C} K) \mathbf{e}(t, z) \\
& +\bar{B} \mathbf{e}(t-\tau, z)+\bar{C} L \mathbf{e}_{z}(t, z)
\end{aligned}
$$

Next, the asymptotical stability will be analyzed for the closed loop system (22) and the controller will be designed.

Theorem 3. For the coupled DPS (19), synchronize to the system (6) if there are matrices $X=X^{T}>0, S=S^{T}>0$, $Y$ and $R$ satisfying the following linear matrix inequalities:

$$
\bar{\Omega}=\left[\begin{array}{ccc}
\bar{A} X+X \bar{A}^{T}+\bar{C} Y+Y^{T} \bar{C}+S & \bar{B} X & \bar{C} R \\
* & -S & 0 \\
* & 0 & -2 \bar{D} X
\end{array}\right]<0,
$$

where $*$ represents blocks that are readily inferred by symmetry. Then the controller gains can be constructed as

$$
K=Y X^{-1}, \quad L=R X^{-1} .
$$

Proof. Consider the following Lyapunov functional candidate for system:

$$
\begin{aligned}
V(t)= & \int_{z_{1}}^{z_{2}} \mathbf{e}^{T}(t, z) P \mathbf{e}(t, z) d z \\
& +\int_{z_{1}}^{z_{2}} \int_{t-\tau}^{t} \mathbf{e}^{T}(\theta, z) Q \mathbf{e}(\theta, z) d \theta d z,
\end{aligned}
$$

where $P=P^{T}>0$ and $Q=Q^{T}>0$. Consider

$$
\begin{aligned}
\frac{d V}{d t}= & 2 \int_{z_{1}}^{z_{2}} \mathbf{e}^{T}(t, z) P \mathbf{e}_{t}(t, z) d z \\
& +\int_{z_{1}}^{z_{2}}\left[\mathbf{e}^{T}(t, z) Q \mathbf{e}(t, z)-\mathbf{e}^{T}(t-\tau, z)\right. \\
& \times Q \mathbf{e}(t-\tau, z)] d z \\
= & 2 \int_{z_{1}}^{z_{2}} \mathbf{e}^{T}(t, z) P\left[\bar{D} \mathbf{e}_{z z}(t, z) d z+(\bar{A}+\bar{C} K) \mathbf{e}(t, z)\right. \\
& \left.+\bar{B} \mathbf{e}(t-\tau, z)+\bar{C} L \mathbf{e}_{z}(t, z)\right] d z \\
& +\int_{z_{1}}^{z_{2}}\left[\mathbf{e}^{T}(t, z) Q \mathbf{e}(t, z)-\mathbf{e}^{T}(t-\tau, z)\right. \\
= & 2 \int_{z_{1}}^{z_{2}} \mathbf{e}^{T}(t, z) P \bar{D} \mathbf{e}_{z z}(t, z) d z \\
& +\int_{z_{1}}^{z_{2}} \mathbf{e}^{T}(t, z)[2 P(\bar{A}+\bar{C} K)+Q] \mathbf{e}(t, z) d z \\
& +\int_{z_{1}}^{z_{2}} \mathbf{e}^{T}(t, z) 2 P \bar{B} \mathbf{e}(t-\tau, z) d z \\
& +\int_{z_{1}}^{z_{2}} \mathbf{e}^{T}(t, z) 2 P \bar{C} L \mathbf{e}_{z}(t-\tau, z) d z \\
& -\int_{z_{1}}^{z_{2}} \mathbf{e}^{T}(t-\tau, z) Q \mathbf{e}(t-\tau, z) d z .
\end{aligned}
$$

From the proof of Theorem 2, we can derive that

$$
\frac{d V}{d t}=\int_{z_{1}}^{z_{2}} \xi^{T}(t, z) \widehat{\Omega} \xi(t, z) d z<0,
$$

where $\xi(t, z)=\left[\mathbf{e}^{T}(t, z), \mathbf{e}^{T}(t-\tau, z), \mathbf{e}_{z}^{T}(t, z)\right]^{T}$

$$
\widehat{\Omega}=\left[\begin{array}{ccc}
P(\bar{A}+\bar{C} K)+(\bar{A}+\bar{C} K)^{T} P+Q & P \bar{B} & P \bar{C} L \\
\bar{B}^{T} P & -Q & 0 \\
L^{T} \bar{C}^{T} P & 0 & -2 P \bar{D}
\end{array}\right]<0 .
$$

Next, we prove that (28) holds if LMIs (23) are satisfied. Pre- and post-Kronecker product $P^{-1}$ to (28), we can derive that $P^{-1} \otimes \widehat{\Omega} \otimes P^{-1}<0$ and apply the change of variables $X=P^{-1}$ and $S=P^{-1} Q P^{-1}$ such that

$$
\bar{\Omega}=\left[\begin{array}{ccc}
\bar{A} X+X \bar{A}^{T}+\bar{C} Y+Y^{T} \bar{C}+S & \bar{B} X & \bar{C} R \\
X \bar{B}^{T} & -S & 0 \\
R^{T} \bar{C} & 0 & -2 \bar{D} X
\end{array}\right]<0,
$$

where $Y=K X$ and $R=L X$. 


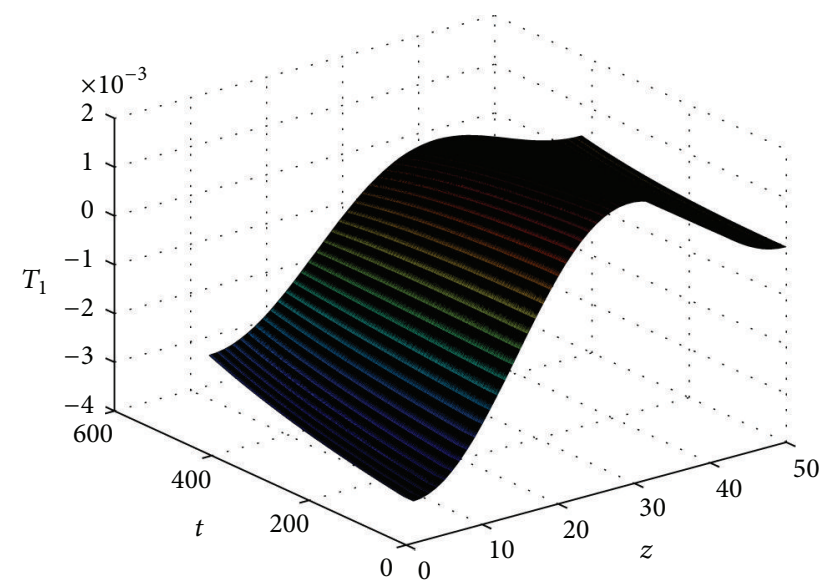

FIGURE 1: State of the first distributed parameter system.

\section{Simulation}

Consider the following distributed parameter system:

$$
\begin{gathered}
\left.\frac{\partial T_{k}}{\partial t}=\frac{\partial^{2} T_{k}}{\partial z^{2}}+\alpha T_{k}(t)+\beta T_{k}(t-\tau)\right) \\
+\sum_{j=1}^{N} G_{k j} \frac{\partial^{2} T_{j}}{\partial z^{2}}, \quad z \in(0, \pi), \\
\left.\frac{\partial T}{\partial z}\right|_{z=0}=0,\left.\quad \frac{\partial T}{\partial z}\right|_{z=\pi}=0, \\
T(t, z)=\cos z+\cos 2 z, \quad t \in[-\tau, 0]
\end{gathered}
$$

where time delay $\tau=1, i=1,2,3$ are the state variable of the $i$ th neural network. Choosing the coupling matrix and coupling matrices as follows:

$$
G=0.01 \times\left[\begin{array}{ccc}
-2 & 1 & 1 \\
1 & -2 & 1 \\
1 & 1 & -2
\end{array}\right]
$$

From Figures 1, 2, and 3, we can derive that $T_{k}(k=1,2,3)$ cannot synchronize. Next the controller will be designed to synchronize three distributed parameter systems.

Let $\alpha=0.01, \beta=-0.02$. Figures $1-3$ give the open-loop state $T_{k}(k=1,2,3)$ responses. As shown in the simulation, we can find that the coupled distributed parameter system can not synchronize partially under $u=0$. From Theorem 3 , the observer and controller gains are constructed via solving the matrix inequalities (23), we obtain the controller gain matrices as follows:

$$
K=\left[\begin{array}{lll}
-1.9706 & -0.0201 & -0.0366 \\
-0.0202 & -1.9705 & -0.0310 \\
-0.0033 & -0.0088 & -1.9701
\end{array}\right]
$$

and $L$ is zero matrix.

Under the designed controller, Figures 4, 5, and 6 show the coupled distributed parameter system synchronization.

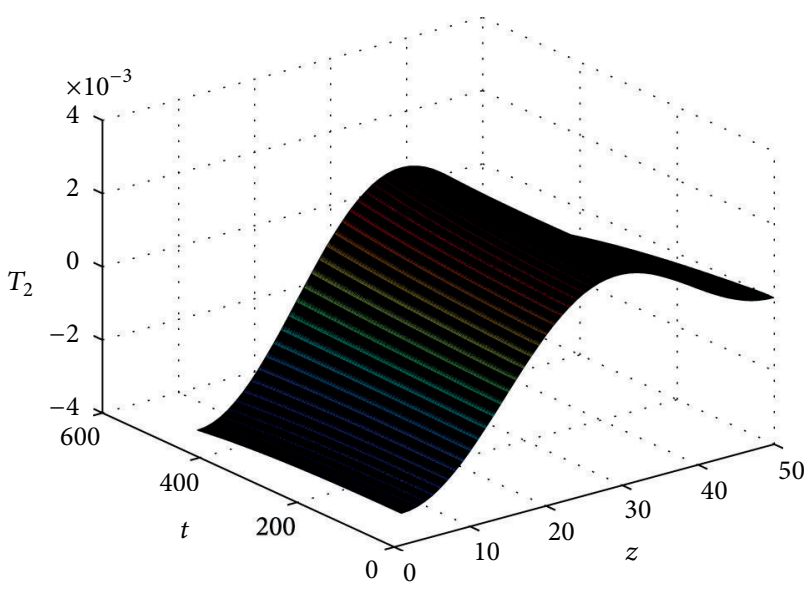

FIGURE 2: State of the second distributed parameter system.

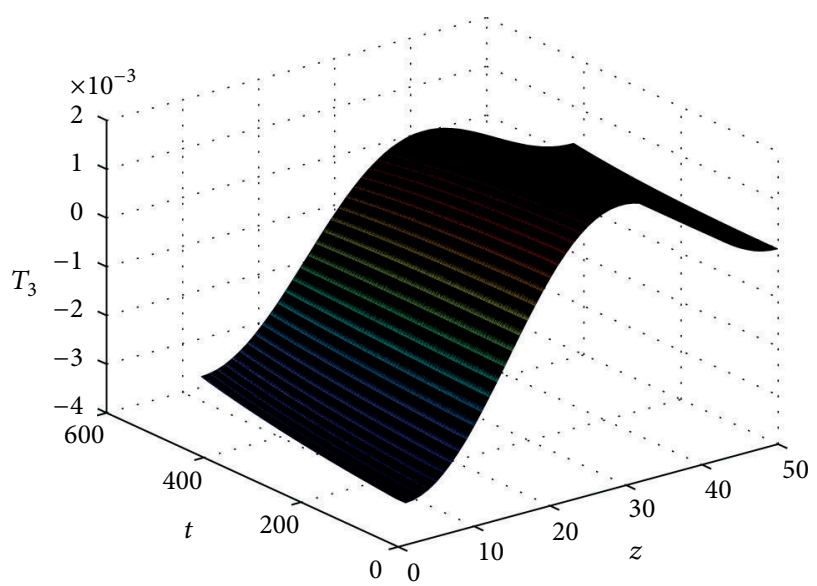

FIGURE 3: State of the third distributed parameter system.

\section{Conclusion}

In this paper, we have addressed the problem of synchronization problem for a class of coupled DPS. First, some sufficient conditions are given to ensure the synchronization of the coupled DPS. Secondly, in the case that the coupled DPS cannot synchronize by itself, the P-sD state feedback control has been designed and applied to force the coupled DPS to synchronize. The controller has been developed in terms of LMIs based directly on the error system. The coupled DPS can synchronize to the isolated node under the controller. Finally, the developed design method is applied to the simulation example, and the achieved simulation results show the effectiveness and benefit of the proposed controller. It is worth pointing out that the proposed design method is easily developed for the coupled DPS with Lipschtiz nonlinear function.

\section{Conflict of Interests}

The authors declare that there is no conflict of interests regarding the publication of this paper. 


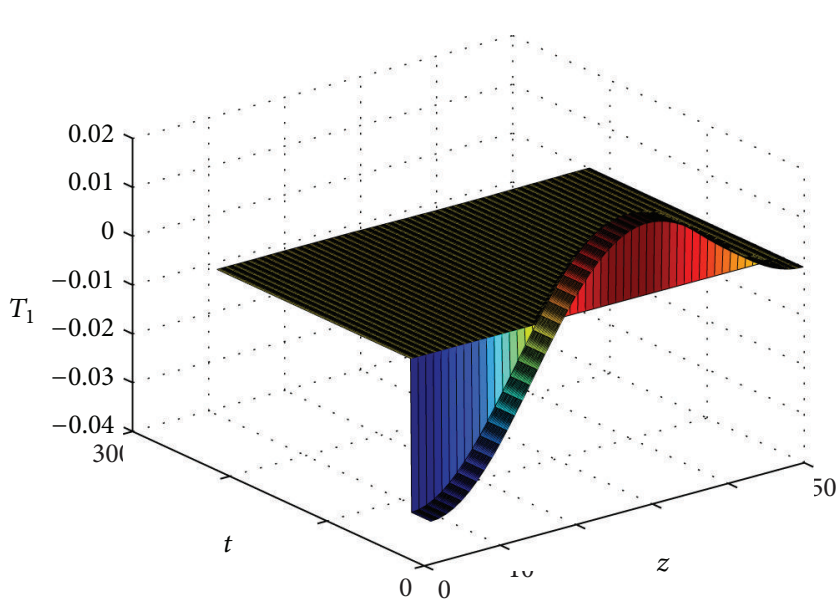

FIGURE 4: State of the first distributed parameter system under the controller.

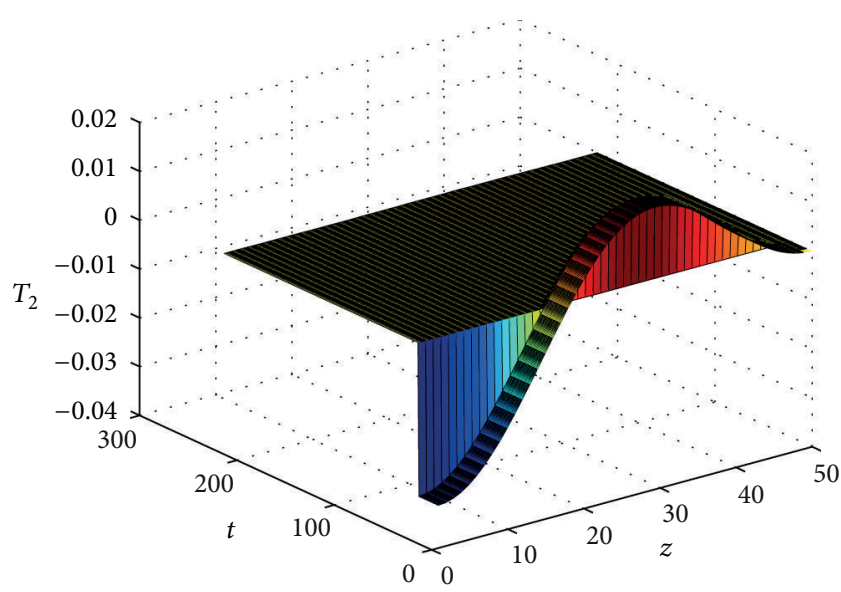

FIGURE 5: State of the second distributed parameter system under the controller.

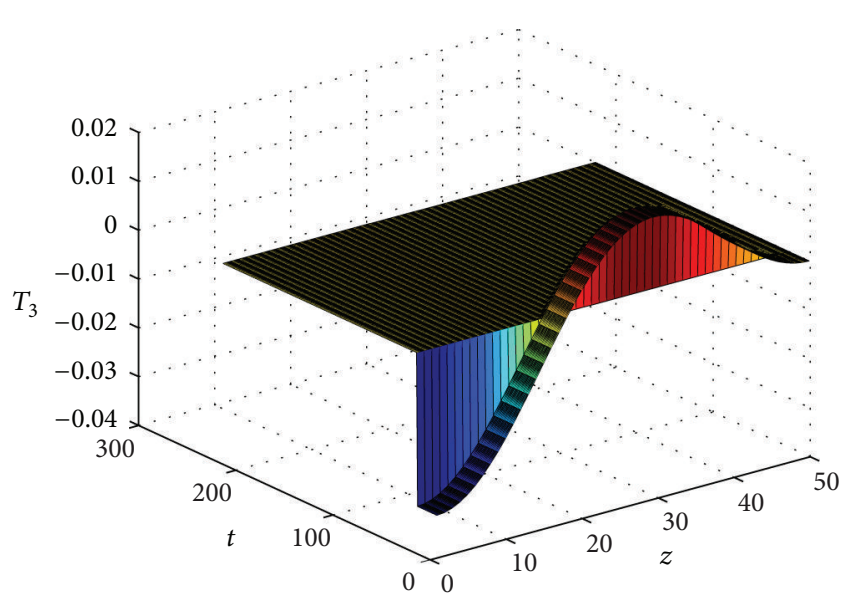

FIGURE 6: State of the third distributed parameter system under the controller.

\section{Acknowledgment}

This work was funded by the Deanship of Scientific Research (DSR), King Abdulaziz University (KAU), under Grant 3130/1434/HiCi. The authors, therefore, acknowledge the technical and financial support of KAU.

\section{References}

[1] J. C. Robinson, "Finite-dimensional behavior in dissipative partial differential equations," Chaos, vol. 5, no. 1, pp. 330-345, 1995.

[2] P. D. Christofides, Nonlinear and Robust Control of PDE Systems: Methods and Applications to Transport-Reaction Processes, Birkhäuser, Boston, Mass, USA, 2001.

[3] H. Deng, H.-X. Li, and G. Chen, "Spectral-approximationbased intelligent modeling for distributed thermal processes," IEEE Transactions on Control Systems Technology, vol. 13, no. 5, pp. 686-700, 2005.

[4] C. Antoniades and P. D. Christofides, "Non-linear feedback control of parabolic partial differential difference equation systems," International Journal of Control, vol. 73, no. 17, pp. 1572-1591, 2000.

[5] H.-X. Li, H. Deng, and J. Zhong, "Model-based integration of control and supervision for one kind of curing process," IEEE Transactions on Electronics Packaging Manufacturing, vol. 27, no. 3, pp. 177-186, 2004.

[6] M. J. Balas, "The Galerkin method and feedback control of linear distributed parameter systems," Journal of Mathematical Analysis and Applications, vol. 91, no. 2, pp. 527-546, 1983.

[7] R. F. Curtain, "Finite-dimensional compensator design for parabolic distributed systems with point sensors and boundary input," IEEE Transactions on Automatic Control, vol. 27, no. 1, pp. 98-104, 1982.

[8] M. A. Erickson, R. S. Smith, and A. J. Laub, "Finite-dimensional approximation and error bounds for spectral systems with partially known eigenstructure," IEEE Transactions on Automatic Control, vol. 39, no. 9, pp. 1904-1909, 1994.

[9] P. D. Christofides and P. Daoutidis, "Finite-dimensional control of parabolic PDE systems using approximate inertial manifolds," Journal of Mathematical Analysis and Applications, vol. 216, no. 2, pp. 398-420, 1997.

[10] P. D. Christofides, "Robust control of parabolic PDE systems," Chemical Engineering Science, vol. 53, no. 16, pp. 2949-2965, 1998.

[11] P. D. Christofides and J. Baker, "Robust output feedback control of quasi-linear parabolic PDE systems," Systems \& Control Letters, vol. 36, no. 5, pp. 307-316, 1999.

[12] J. Baker and P. D. Christofides, "Finite-dimensional approximation and control of non-linear parabolic PDE systems," International Journal of Control, vol. 73, no. 5, pp. 439-456, 2000.

[13] K. A. Hoo and D. Zheng, "Low-order control-relevant models for a class of distributed parameter systems," Chemical Engineering Science, vol. 56, no. 23, pp. 6683-6710, 2001.

[14] H. M. Park and D. H. Cho, "The use of the KarhunenLoève decomposition for the modeling of distributed parameter systems," Chemical Engineering Science, vol. 51, no. 1, pp. 81-98, 1996.

[15] B.-S. Chen and Y.-T. Chang, "Fuzzy state-space modeling and robust observer-based control design for nonlinear partial 
differential systems," IEEE Transactions on Fuzzy Systems, vol. 17, no. 5, pp. 1025-1043, 2009.

[16] K. Yuan, S.-M. Fei, and J.-D. Cao, "Partial synchronization of the distributed parameter system with time delay via fuzzy control," IMA Journal of Mathematical Control and Information, 2013.

[17] J.-W. Wang, H.-N. Wu, and H.-X. Li, "Distributed proportionalspatial derivative control of nonlinear parabolic systems via fuzzy PDE modeling approach," IEEE Transactions on Systems, Man, and Cybernetics B, vol. 42, no. 3, pp. 927-938, 2012.

[18] Y.-T. Chang and B.-S. Chen, "A fuzzy approach for robust reference-tracking-control design of nonlinear distributed parameter time-delayed systems and its application," IEEE Transactions on Fuzzy Systems, vol. 18, no. 6, pp. 1041-1057, 2010.

[19] J.-W. Wang, H.-N. Wu, and H.-X. Li, "Distributed fuzzy control design of nonlinear hyperbolic pde systems with application to nonisothermal plug-flow reactor," IEEE Transactions on Fuzzy Systems, vol. 19, no. 3, pp. 514-526, 2011.

[20] K. Yuan, H.-X. Li, and J. Cao, "Robust stabilization of the distributed parameter system with time delay via fuzzy control," IEEE Transactions on Fuzzy Systems, vol. 16, no. 3, pp. 567-584, 2008.

[21] C. W. Wu and L. O. Chua, "Synchronization in an array of linearly coupled dynamical systems," IEEE Transactions on Circuits and Systems I, vol. 42, no. 8, pp. 430-447, 1995.

[22] C. W. Wu, "Synchronization in coupled arrays of chaotic oscillators with nonreciprocal coupling," IEEE Transactions on Circuits and Systems I, vol. 50, no. 2, pp. 294-297, 2003.

[23] C. Li and G. Chen, "Synchronization in general complex dynamical networks with coupling delays," Physica A, vol. 343, no. 1-4, pp. 263-278, 2004.

[24] E. M. Izhikevich and F. C. Hoppensteadt, "Slowly coupled oscillators: phase dynamics and synchronization," SIAM Journal on Applied Mathematics, vol. 63, no. 6, pp. 1935-1953, 2003.

[25] J. Lü and G. Chen, "A time-varying complex dynamical network model and its controlled synchronization criteria," IEEE Transactions on Automatic Control, vol. 50, no. 6, pp. 841-846, 2005.

[26] W. Lu and T. Chen, "New approach to synchronization analysis of linearly coupled ordinary differential systems," Physica D. Nonlinear Phenomena, vol. 213, no. 2, pp. 214-230, 2006.

[27] K. Wu and B.-S. Chen, "Synchronization of partial differential systems via diffusion coupling," IEEE Transactions on Circuits and Systems I, vol. 59, no. 11, pp. 2655-2668, 2012. 


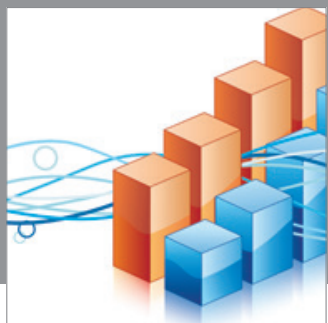

Advances in

Operations Research

mansans

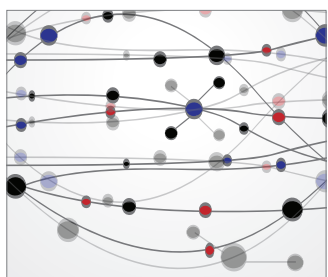

The Scientific World Journal
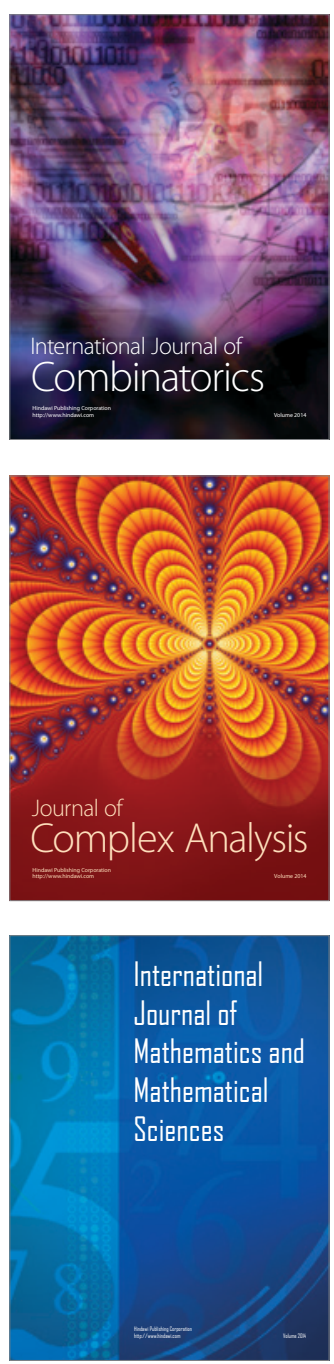
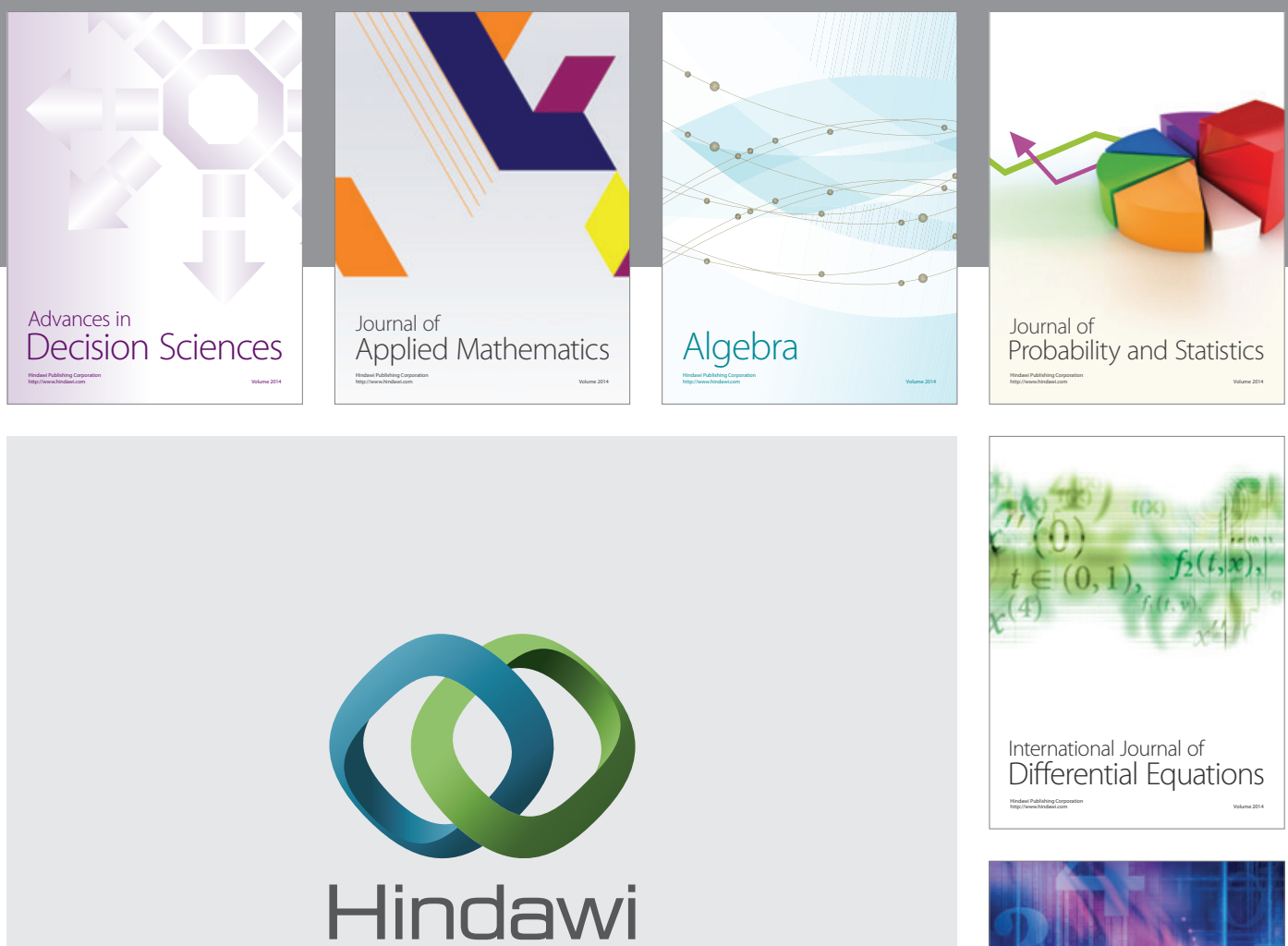

Submit your manuscripts at http://www.hindawi.com
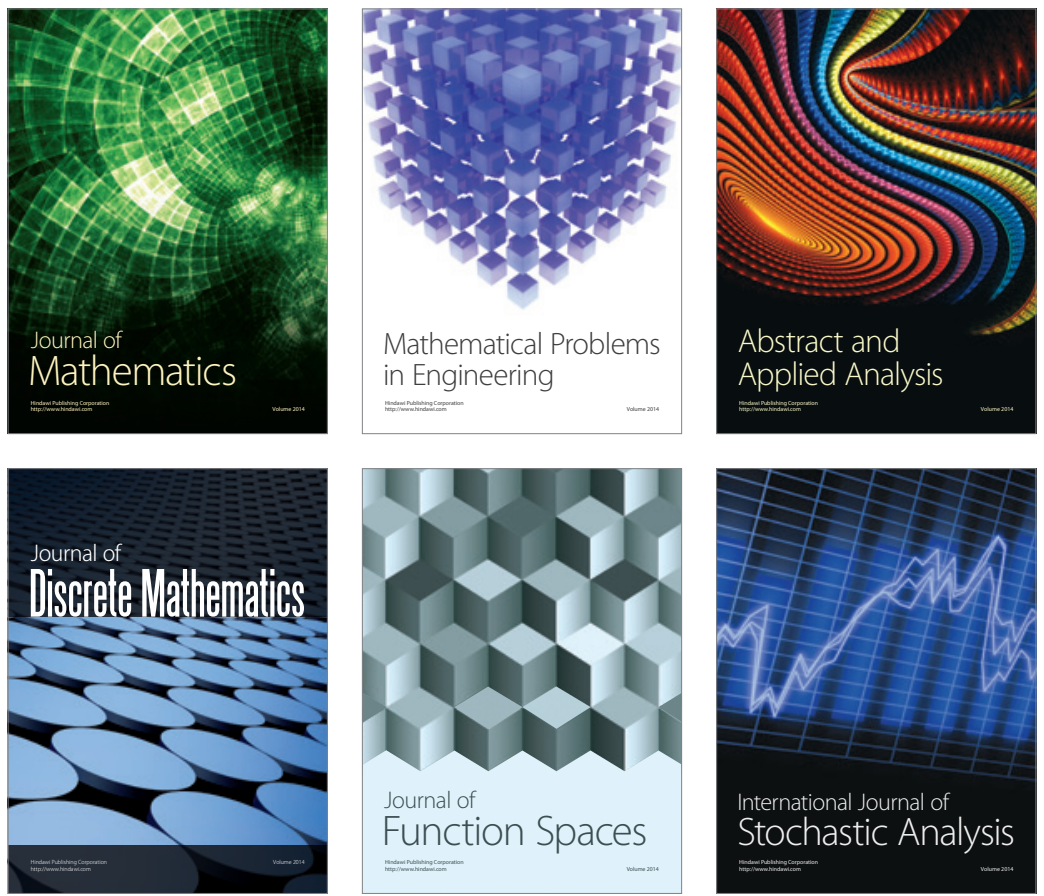

Journal of

Function Spaces

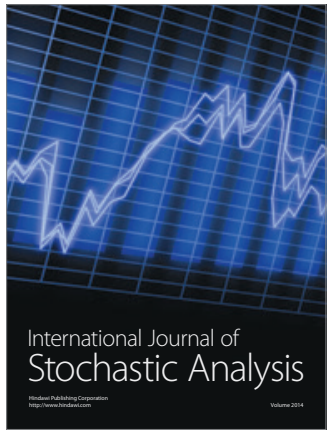

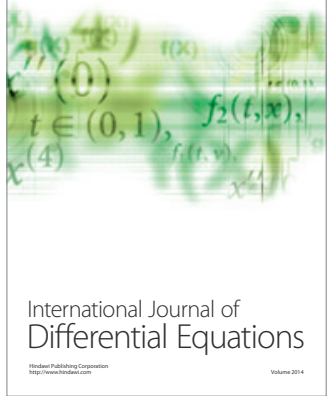
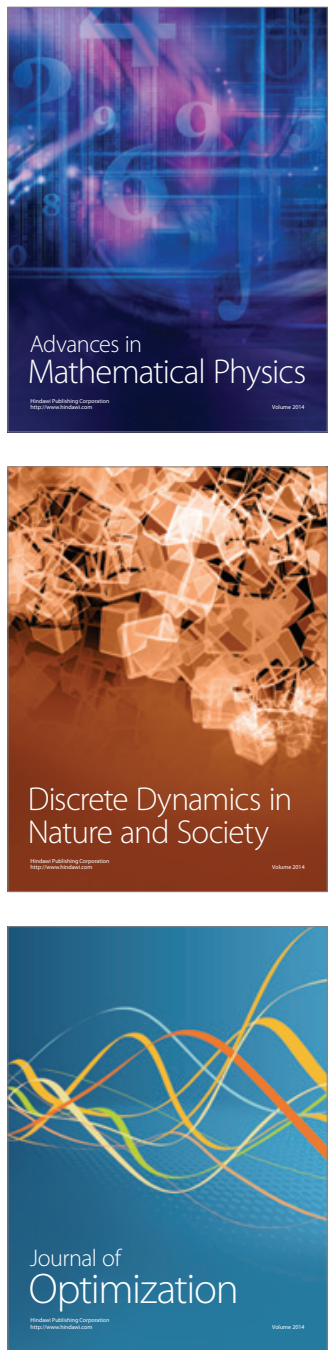